\title{
Population spread and cultural transmission in Neolithic transitions
}

\author{
Joaquim Fort \\ Complex Systems Lab and Physics \\ Department, University of Girona, \\ C/. Ma Aurèlia Capmany 61, 17071 \\ Girona, Catalonia, Spain. \\ Email: joaquim.fort@udg.edu
}

\author{
Neus Isern \\ Laboratori d'Arqueologia \\ Quantitativa (LAQU), \\ Departament de Prehistòria, \\ Universitat Autònoma de \\ Barcelona, 08193 Cerdanyola del \\ Vallès, Spain. \\ Email: neus.isern@uab.cat
}

\author{
Antonieta Jeradino, \\ Bernardo Rondelli \\ ICREA/CaSEs Research Group, \\ Department of Archaeology and \\ Anthropology, Institució Milà i \\ Fontanals, CSIC, C/. Egipcíaques, \\ 15, 08001 Barcelona, Spain. \\ Email: a.jerardino@imf.csic.es
}

\begin{abstract}
The classical wave-of-advance model is based on Fisher's equation. However, this approach leads to an unbounded wave-of-advance speed at high reproduction rates. In contrast, an integro-difference model leads to a finite upper bound for the speed, namely the maximum dispersal distance divided by the generation time. Intuitively, this is a very reasonable result. This demic model has been generalized to include cultural transmission (Fort, PNAS 2012). We apply this recent demic-cultural model to determine the percentages of demic and cultural diffusion in the Neolithic transition for two case studies: (i) Europe, and (ii) southern Africa (Jerardino et al., submitted 2014). The similarities and differences between both case studies are interpreted in terms of the three mechanisms at work (population reproduction, dispersal and acculturation).
\end{abstract}

\section{INTRODUCTION}

$\mathrm{T}$ HE Neolithic transition in Europe has been analyzed quantitatively since the seminal work by Ammerman and Cavalli-Sforza [1]. Because the oldest Neolithic sites are located in the Near East, Ammerman and Cavalli-Sforza [1] fitted a straight line to the dates of European sites versus their distances to a Near Eastern site (Jericho). In this way they estimated a speed of about $1 \mathrm{~km} / \mathrm{y}$. Later Ammerman and Cavalli-Sforza [2,3] applied a model due to Fisher [4] to the spread of preindustrial famers. They found that this model predicts a speed of about $1 \mathrm{~km} / \mathrm{y}$, i.e. similar to the observed one. This indicates that a process based mainly on demic diffusion (spread of populations) agrees with the archaeological data in Europe. Here we report on models with a more refined description of population spread than Fisher's model $[5,6]$. We also recall a recent model that incorporates the effect of cultural diffusion, i.e. the spread of ideas (hunter-gatherers becoming farmers) instead of populations [7]. This demic-cultural model is then compared to the archaeological data on the Neolithic spread in Europe and southern Africa.

This work was partially funded by the MINECO projects SimulPast (Consolider-CSD2010-00034, all authors), FIS-2009-13050 and FIS-201231307 (JF) and the Generalitat de Catalunya project 2014-SGR-36 (JF).

\section{LIMITATIONS OF FISHER'S MODEL}

Consider a population of preindustrial farmers, initially located in some region. Assume they can disperse into other regions that are also suitable for farming but initially empty of farmers. The next generations of farmers will, in general, disperse away from their parents. Then Fisher's model predicts that a wave of advance (also called a front) of farmers will form and propagate with the following speed [4]

$$
S_{F}=2 \sqrt{a_{N} D_{N}},
$$

where $a_{N}$ is the initial reproduction rate of Neolithic farmers (which is easily related to their net fecundity and generation time) and $D_{N}$ is the diffusion coefficient of Neolithic farmers (which is easily related to the probability that farmers disperse away from their parents as a function of distance). Equation (1) is very useful. Ammerman and Cavalli-Sforza [2,3] used observed values for $a_{N}$ and $D_{N}$ into Eq. (1) and found that Fisher's model predicts a speed of about $1 \mathrm{~km} / \mathrm{y}$, i.e. similar to the observed one for the Neolithic transition in Europe.

In recent years, Fisher's model has been refined [5]. Note that Eq. (1) predicts that, for a given value of $D_{N}$, the speed increases without bound $\left(s_{F} \rightarrow \infty\right)$ for increasing values of the initial reproduction rate $\left(a_{N} \rightarrow \infty\right)$. This is counterintuitive because, for a given value of $D_{N}$, the dispersal behavior of the population is fixed. Thus individuals can disperse up to some maximum distance, $\Delta_{\max }$. Then we should expect that (no matter how large is $a_{N}$ ) the speed $s_{F}$ should not be faster than $s_{\max }=\Delta_{\max } / T$, where $T$ is the time interval between two subsequent migrations (mean age difference between parents and their children). An integro-difference cohabitation model solves this problem [5-7]. Then Eq. (1) is replaced by a more complicated and accurate equation. However Fisher's speed, Eq. (1), is very useful as a first approximation. It is even 
quite accurate for some pre-industrial farming populations. For example, for the Yanomano [8] Fisher's speed (1.22 $\mathrm{km} / \mathrm{y}$ ) yields an error of only $6 \%$ relative to the integrodifference cohabitation model $(1.30 \mathrm{~km} / \mathrm{y})$. In other cases, Fisher's speed is not so accurate. For example, for the Issocongos [8] Fisher's speed $(0.56 \mathrm{~km} / \mathrm{y})$ yields an error of $30 \%$ relative to the integro-difference cohabitation model $(0.80 \mathrm{~km} / \mathrm{y})$.

\section{POSSIBLE FORMS OF THE CULTURAL TRANSMISSION TERM}

The demic models above can be extended by including cultural transmission. Then Fisher's speed, Eq. (1) is generalized into [7]

$$
s=2 \sqrt{\left(a_{N}+\frac{C}{T}\right) D_{N},}
$$

where $C$ is the intensity of cultural transmission (defined as the number of hunter-gatherers converted into farmers per farmer during his/her lifetime, in the leading edge of the front, i.e. a region where the population density of farmers is very low) [7]. In the absence of cultural transmission $(C=0)$, Eq. (2) reduces to Fisher's speed, Eq. (1), as it should.

Equation (2) and other models with cultural transmission take into account that hunter-gatherers can learn agriculture not only from incoming farmers, but also from converted hunter-gatherers, i.e. former hunter-gatherers that have (partially) become farmers (as well as their descendants).

An integro-difference cohabitation model with cultural transmission leads to a more complicated equation than Eq. (2), and generalizes the integro-difference model summarized in the previous section [7].

Both demic-cultural models (i.e., Eq. (2) and the integrodifference cohabitation model) are based on cultural transmission theory [9], which shows that the number of hunter-gatherers converted into farmers per farmer during his/her lifetime is [7]

$$
\frac{\Delta P_{N}}{P_{N}}=\frac{f P_{P}}{P_{N}+\gamma P_{P}},
$$

where $P_{N}$ and $P_{P}$ are the population densities of Neolithic farmers and Mesolithic hunter-gatherers, respectively, and $f$ and $\gamma$ are cultural transmission parameters. In the leading edge of the front $\left(P_{N} \approx 0\right)$, Eq. (3) becomes

$$
\frac{\Delta P_{N}}{P_{N}}=C,
$$

with $C=f / \gamma$.

A comparison to other approaches is of interest here. In Ecology a widely used model is based on Lotka-Volterra equations, which assume that the interaction between two populations $\left(\Delta P_{N}\right)$ is proportional to their population densities [10],

$$
\frac{\Delta P_{N}}{P_{N}}=k P_{P},
$$

where $k$ is a constant. This model has the problem that $\Delta P_{N} / P_{N} \rightarrow \infty$ if $P_{P} \rightarrow \infty$, which seems inappropriate in cultural transmission, for the following reason. Assume that a farmer converts, e.g., 5 hunter-gatherers during his lifetime $\left(\Delta P_{N} / P_{N}=5\right)$ if there are $P_{P}=10$ hunter-gatherers per unit area. Then Eq. (5) predicts that he/she will convert $\Delta P_{N} / P_{N}=50$ hunter-gatherers if there are $P_{P}=100$ huntergatherers per unit area, $\Delta P_{N} / P_{N}=500$ hunter-gatherers if there are $P_{P}=1000$ hunter-gatherers per unit area, etc. Contrary to this, intuitively we expect that there should be a maximum in the number of hunter-gatherers that a famer can convert during his/her lifetime, i.e. that $\Delta P_{N} / P_{N}$ should have a finite limit if $P_{P} \rightarrow \infty$. This saturation effect is indeed predicted by Eq. (3), as shown by Eq. (4). Thus we think that Eq. (3) is more reasonable than the Lotka-Volterra interaction, Eq. (5).

This point has important consequences because for Eq. (3) the wave-of-advance speed is independent of the carrying capacity of hunter-gatherers, $P_{P \max }$ (see, e.g., Eq. (2)). In contrast, for the Lotka-Volterra interaction the waveof-advance speed does depend on $P_{P \max }$. For example, if

Fisher's model is generalized by including the LotkaVolterra interaction, the front speed is [11] (see also [10] for a similar model)

$$
s=2 \sqrt{\left(a_{N}+\frac{k P_{P \max }}{T}\right) D_{N} .}
$$

The point is that, in contrast to Eq. (2), Eq. (6) depends on $P_{P \max }$. The same happens if the integro-difference cohabitation model (which is more precise than Fisher's model) is generalized by including the Lotka-Volterra interaction [6]. These results are not surprising because in the front leading edge $\left(P_{N} \approx 0, P_{P} \approx P_{P \max }\right)$ Eq. (5) becomes $\Delta P_{N} / P_{N}=k P_{P \max }$, which depends on $P_{P \max }$ (whereas Eq. (4) does not).

Finally, some language competition models use population fractions (rather than population densities) and interaction terms with non-linear powers of $P_{N}$ and $P_{P}$ [12]. We first consider the linear case. In one such model, Eq. (5) above is replaced by [13]

$$
\frac{\Delta P_{N}}{P_{N}}=\frac{\eta P_{P}}{P_{N}+P_{P}},
$$

with $\eta$ a constant. Equation (7) is a special case of Eq. (3), thus the wave-of-advance speed is independent of $P_{P \max }$ also in this model [13]. It can be argued that the complete model in Ref. [13] is useful for modern populations but not 
for the Neolithic transition, because it assumes the same carrying capacity for both populations. But a model that allows for different carrying capacities [14] also leads, in the linear case, to an equation with the form of Eq. (7). In conclusion, some models originally devised to describe language competition also lead to the conclusion we have stressed above, namely that the wave-of-advance speed is independent of $P_{P \max }$.

For completeness, in the non-linear case the following two limitations of the language-competition models discussed in the previous paragraph [12-14] should be noted in the context of the Neolithic transition.

(i) In the non-linear case, Eq. (7) above is generalized into [13]

$$
\Delta P_{N}=\frac{\eta P_{N}^{\alpha} P_{P}^{\beta}}{\left(P_{N}+P_{P}\right)^{\alpha+\beta-1}}
$$

with $\alpha \geq 1$ and $\beta \geq 1$ [12]. Thus $\Delta P_{N} \rightarrow 0$ if $P_{P} \rightarrow \infty$, i.e. $\Delta P_{N} / P_{N}$ does not have a finite, non-vanishing limit (except in the linear case $\alpha=\beta=1$, see Eq. (6)). Alternatively, for the Abrams-Strogatz model in Ref. [14], namely

$$
\Delta P_{N}=\kappa\left[\sigma P_{P}\left(\frac{P_{N}}{P_{N}+P_{P}}\right)^{a}-(1-\sigma) P_{N}\left(\frac{P_{P}}{P_{N}+P_{P}}\right)^{a}\right],
$$

where $\sigma<1$ is called the status of language $\mathrm{N}$ and $a \geq 1$ is the resistance to language change, we obtain a negative limit for $\Delta P_{N} / P_{N}$ if $P_{P} \rightarrow \infty$, which is counterintuitive [13] (except again in the linear case, $a=1$ ). The main point here is that neither of both non-linear models displays the saturation effect discussed above.

(ii) Whereas Eq. (3) was derived from cultural transmission theory, the non-linear models introduced to describe language competition [12-14] (Eqs. (8)-(9)) were not.

The non-linear models given by Eqs. (8)-(9) compare favorably to observed data in non-spatial linguistic systems $[12,13]$, and may be applicable to other modern instances of cultural transmission. Perhaps the effects of mass-media, schools, etc. in modern societies avoid the saturation effect discussed above. Such effects are not included in the cultural transmission theory leading to Eq. (3) [7].

In any case, due to reasons (i) and (ii) above, for the Neolithic transition we prefer not to apply languagecompetition non-linear models, Eqs. (8)-(9), neither the Lotka-Volterra interaction, Eq. (5). Instead, we apply cultural transmission theory, Eq. (3) (or its frequencydependent generalizations, which take into account the conformist effect but lead to the same conclusions [7]).

We stress that the conclusion that the wave-of-advance speed is independent of the hunter-gatherer population density $P_{P \max }$ follows from cultural transmission theory, and is ultimately due to the fact that there should be a maximum number of hunter-gatherers converted to agriculture per farmer (or converted hunter-gatherer) during his/her lifetime (this is the saturation effect discussed above).

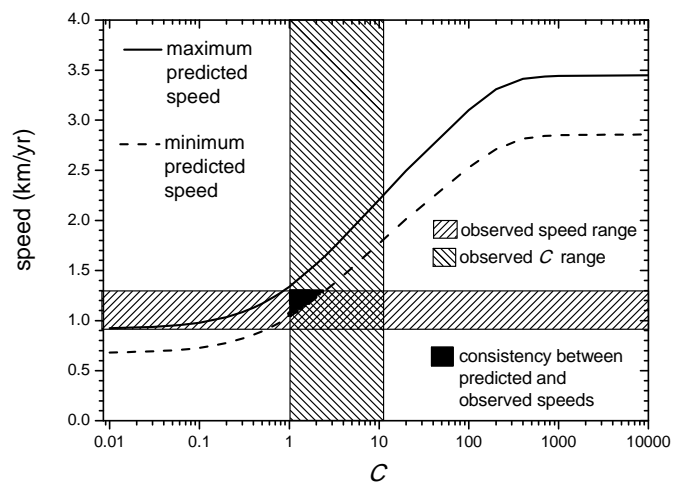

Fig. 1 The speed of the Neolithic transition, as a function of the intensity of cultural transmission $C$. The horizontal hatched rectangle is the observed speed range of the Neolithic transition in Europe. Adapted from Ref. [7]

\section{EUROPE}

The integro-difference cohabitation model that refines Eq. (2) has been applied to the Neolithic transition in Europe [7]. The results are reproduced in Fig. 3. Note that without taking into account the effect of cultural transmission $(C=0)$, the predicted speed is about $0.8 \mathrm{~km} / \mathrm{y}(0.7-0.9$ $\mathrm{km} / \mathrm{y}$ ), whereas for consistent values of $C$ the speed increases up to $1.3 \mathrm{~km} / \mathrm{y}$. Thus the cultural effect is about $40 \%$ (more precisely, $40 \pm 8 \%$ [7]).

\section{SOUTHERN AFrICA}

In this case the Neolithic transition was a shift from hunting-gathering into herding (not into farming and stockbreeding as in Europe), the speed is substantially faster than in the European case and, in agreement with Fig. 1, the cultural effect is more important [15].

\section{CONCLUSION}

We have discussed wave-of-advance models of the spread of the Neolithic under demic and/or cultural diffusion. Such models lead to the conclusion that this spread was mainly demic in Europe, but mainly cultural in southern Africa. Because the reproductive and dispersal behavior of both populations was likely similar [15], this difference could be due to a higher ease for hunter-gatherers to learn herding in comparison with farming [15].

\section{ACKNOWLEDGMENT}

The authors are very thankful to John Kinahan, Jayson Orton, Thembi Russell, Karim Sadr, and Lita Webley for providing useful bibliography. 


\section{REFERENCES}

[1] A. J. Ammerman, L. L. Cavalli-Sforza, "Measuring the rate of spread of early farming in Europe", Man, vol. 6, pp. 674-688, 1971.

[2] A. J. Ammerman, L. L. Cavalli-Sforza, "A population model for the diffusion of early farming in Europe, C. Renfrew, Ed., The explanation of culture change: models in prehistory. London: Duckworth, 1973, pp. 343-357.

[3] A. J. Ammerman, L. L. Cavalli-Sforza, The Neolithic transition and the genetics of populations in Europe. Princeton (NJ): Princeton Univ. Press, 1984.

[4] R. A. Fisher, "The wave of advance of advantageous genes", Ann. Eugenics, vol. 7, pp. 355-369, 1937.

[5] J. Fort, J. Pérez-Losada, N. Isern, "Fronts from integrodifference equations and persistence effects on the Neolithic transition", Phys. Rev. E, vol. 76, paper 031913, 2007.

[6] J. Fort, J. Pérez-Losada, J. J. Suñol, L. Escoda, J. M. Massaneda, "Integro-difference equations for interacting species and the Neolithic transition", New J. Phys., vol. 2008, paper 043045, 2008.

[7] J. Fort, "Synthesis between demic and cultural diffusion in the Neolithic transition in Europe", Proc. Natl. Acad. Sci. USA, vol. 109, pp. 18669-18673, 2012.
[8] N. Isern, J. Fort, J. Pérez-Losada, Realistic dispersion kernels applied to cohabitation reaction-dispersion equations. J Stat Mec, vol. 10, paper P10012, 2008.

[9] L. L. Cavalli-Sforza, M. Feldman, Cultural transmission and evolution: A quantitative approach. Princenton: Princeton University Press, 1981.

[10] J. D. Murray, Mathematical Biology, vol. II. Berlin: Springer, 2003, Eqs. (1.2) and (1.10)

[11] V. Méndez, J. Fort, J. Farjas, "Speed of wave-front solutions to hyperbolic reaction-diffusion equations", Phys. Rev. E, vol. 60, pp. 5231-5243, Eq. (50).

[12] D. M. Abrams, S. H. Strogatz, "Modelling the dynamics of language death", Nature, vol. 424, p. 900, 2003.

[13] N. Isern, J. Fort, "Language extinction and linguistic fronts". J. Roy. Soc. Interface, vol. 11, paper 20140028, 2014, Eq. (2.6). Equation (7) here corresponds to the linear case, namely $\alpha=\beta=1$.

[14] J. Fort, J. Pérez-Losada, "Front speed of language replacement", Human Biology, vol. 84, pp. 755-772, 2012.

[15] A. Jerardino, J. Fort, N. Isern and B. Rondelli, "Cultural diffusion was the main driving mechanism of the Neolithic transition in southern Africa", submitted for publication. 